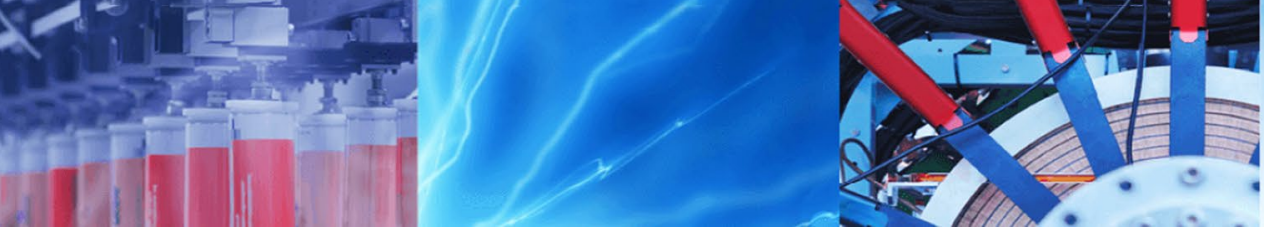

Research Article

\title{
RVE modelling of short fiber reinforced thermoplastics with discrete fiber orientation and fiber length distribution
}

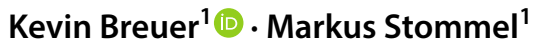

Received: 3 September 2019 / Accepted: 10 December 2019 / Published online: 16 December 2019

(C) The Author(s) 2019 OPEN

\begin{abstract}
This study presents an analysis of modelling aspects on the effective composite properties of short glass fiber reinforced thermoplastics using representative volume elements (RVE). Although, many investigations were published showing effects of different modelling parameters of RVEs, we further elaborate in this contribution the parameters: influence of fiber packing, fiber shape, bonding of the fibers to the matrix, fiber length distribution and fiber orientation. The knowledge of these influences is used to determine the extent to which the increased modelling accuracy and thus the computational effort leads to an improved RVE's forecast quality. This objective is achieved by creating and comparing different RVE models of a PBT-GF20 composite. The information required for the RVE models is obtained by experimental characterization of the PBT-GF20 and the PBT matrix material. It can be concluded based on the results of the numerical investigations in conjunction with the experimental tests of the composite that fiber packing, fiber length distribution, fiber orientation and fiber geometry are essential for a precise determination of the effective composite properties.
\end{abstract}

Keywords RVE · Short fiber reinforced · Fiber geometry · Fiber orientation · Homogenization

\section{Introduction}

The mechanical properties of the short fiber-reinforced plastics depend on a number of factors i.e. on the length and on the orientation of the fibers. Due to the injection molding process, these two factors vary spatially in an injection-molded part, resulting in locally varying composite properties. For an exact dimensioning and design of a technical lightweight application, the spatial distribution of composite properties must be known. These can be calculated by different theories. From the first approaches of Reuss [1] and Voigt [2] up to the commonly two-stage homogenization method [3], the information content about the microstructure, which is included in the calculation of the effective composite properties, increases. This increasing information content is the basis for the increasing forecasting quality of the effective composite properties. Accordingly, efforts are obvious to further increase the forecasting quality by further increasing the information content that is used to calculate the effective composite properties. In this context, the term representative volume elements (RVE) is well known. Hill [4] uses the term RVE for sections of a composite that are statistically representative for the entire composite. According to Hill, the number of inclusions must be sufficiently high so that the calculated composite properties are independent of the selected boundary conditions. Drugan and Willis [5] give a further definition of the term RVE: They define an $\mathrm{RVE}$ as the smallest volume with which an effective mean value of a composite property can be calculated. Gitman et al. [6] work out the existence respectively the nonexistence of RVEs depending on the used constitutive equations as well as the influence of size and periodicity on the effective composite properties on the basis of 2

Kevin Breuer, Kevin.Breuer@tu-dortmund.de | ${ }^{1}$ Mechanical Engineering, Chair of Plastics Technology, TU Dortmund University, Leonhard-Euler-Str. 5, 44227 Dortmund, Germany. 
dimensional RVEs with circular inclusions. It has also been shown in the literature that an effective mean value can be calculated over several small RVE's rather than over a large one [7-9]. Especially Monte-Carlo simulations can be used, in which the realization of a single RVE is randomly generated [10].

The advantage of using RVE's instead of micromechanical models is that less assumptions and simplifications of the underlying microstructure have to be made. This has the advantage that arbitrary shapes of inclusions or an influence of inclusions on each other can be considered in RVEs in contrast to the micromechanical models using homogenization techniques. But, solving RVEs is generally very time-consuming and numerical expensive.

The central question is therefore which information must be at least contained in a RVE to achieve a reasonable representation of the composite properties. Therefore, it is the motivation for this paper to investigate this question by an investigation of the influence of the modelling aspects of short fiber reinforced plastics on the effective composite properties. For this special case in the field of applied material- and engineering science, the influence of modeling aspects has not yet been fully investigated in the known literature. So far, the influence of modeling aspects has been investigated in recently published publications: Wang and Smith, for example, use a parameter study of a unit cell to determine the influence of fiber volume fraction, aspect ratio and distance between the fibers on the effective composite properties [11]. The influence of fiber orientation and fiber volume fraction is investigated in [12, 13]. Furthermore, Chen et al. investigates the composite properties with randomly oriented fibers in all spatial directions in $[14,15]$. The distance between fibers as well as the influence of perpendicular fiber patterns are investigated by Pan et al. [16]. The influence of bent or more specifically kinked fibers is also investigated by Pan et al. in another study [17]. The dependence of linear-viscoelastic properties with respect to the fiber volume fraction is considered in [18]. Wang and his co-authors investigate the effect of an considered interphase between fiber and matrix material in [19]. However, some modelling aspects remain unconsidered despite the known works.

Therefore, in this study further modelling aspects of short fiber reinforced composites, that have not been considered so far, are investigated. In addition, not only individual modeling aspects are examined, but also the combination of different modeling aspects. These modelling aspects are depicted in Fig. 1 and include the fiber packing, meaning the influence of the distance of fibers from each other, the geometry of the fibers as well as the bonding of the fibers to the matrix. The effect of a fiber length distribution in contrast to a homogenous fiber length is also investigated as well as the effect of a fiber orientation in contrast to a perfect alignment of fibers. The modelling aspects mentioned are analyzed individually as well as in combination with each other.

The investigation is carried out using PBT-GF20, exemplarily. Several RVE's considering different modelling aspects are created and evaluated regarding their effective composite stiffness. The aim of this study is to identify the influence of these modelling aspects and to conclude which of them should be modelled with particular precision in order to achieve the best possible RVE's forecast quality results.
Fig. 1 Visualization of the considered modeling aspects with constant fiber volume fraction

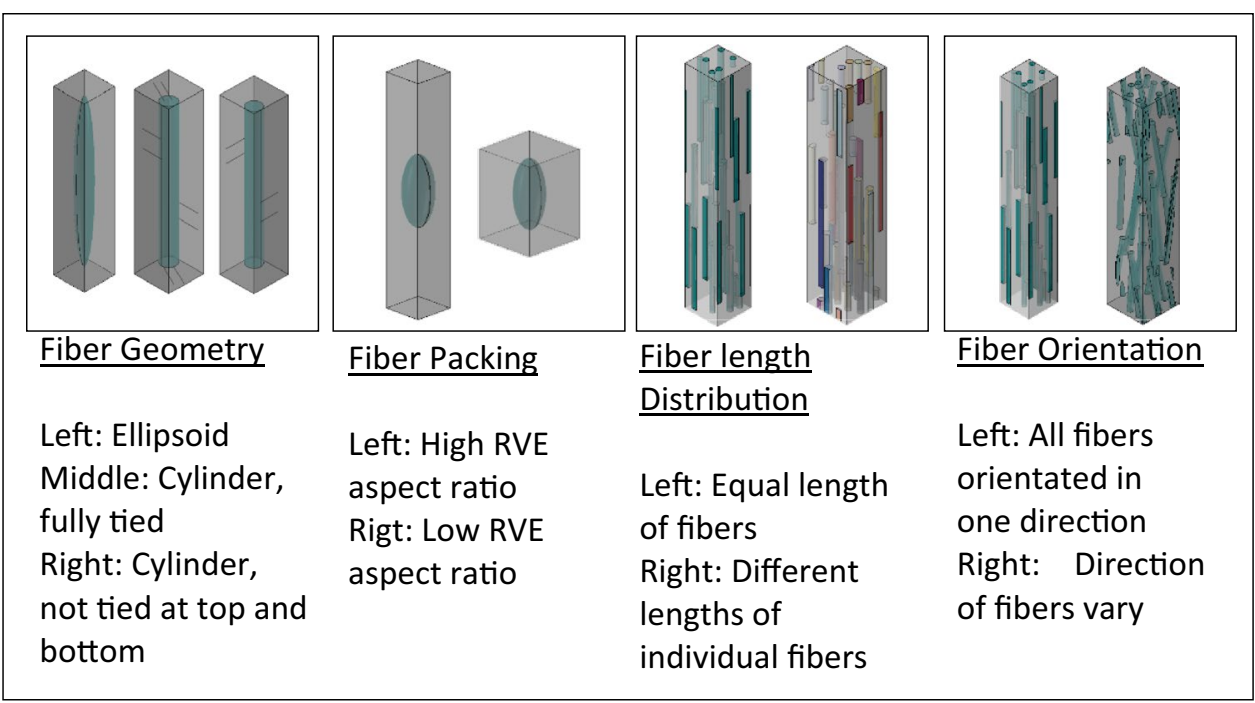




\section{Modelling of RVE}

In this section the geometric definition and evaluation of the RVE's using the Finite Element Method (FEM) is presented. In principle, an RVE is created on the basis of certain modeling aspects, as shown in Fig. 2. In the context of this contribution these modeling aspects comprise the dimension of the RVE, the fiber geometry, the fiber volume fraction, the fiber orientation and the fiber length distribution. The mechanical properties of each phase are also required. In the following, the procedure for creating an RVE is explained in more detail.

First, the dimension and volume of the RVE $V_{R V E}$ must be determined. The volume results from the fiber volume fraction $v_{F}$ and the Volume of all fibers $V_{F}$ to be considered:

$V_{R V E}=V_{F} / V_{F}$.

The dimension $\mathbf{X}_{\mathrm{RVE}}=\left(I_{\text {RVE }}, w_{\text {RVE }}, w_{R V E}\right)$ result from the volume and an aspect ratio $a_{R V E}$ of length $I_{R V E}$ to width $w_{R V E}$ of the RVE, assuming a quadratic base area:

$a_{\text {RVE }}=I_{\text {RVE }} / w_{\text {RVE }}$.

Since the aspect ratio of the RVE influences the fiber packing, this is the first modeling aspect to be investigated in Sect. 4.1.
According to Hill's definition, RVE's must be independent of boundary effects. This can be achieved by creating the RVE large enough so that the boundary effects are negligible. A more efficient approach is to use periodic boundary conditions $[20,21]$. For this purpose, the RVE has to be created in such a way that a periodic fiber constellation is generated.

To create a RVE, first the fibers are considered, whereby the length and the orientation of $n$ fibers are derived from the fiber length distribution and the fiber probability orientation. The $n$ fibers are then placed one by one in the volume. The position of the fibers are randomly selected and periodically continued. This means that fibers that extend the boundary of the RVE are cut off and continued on the opposite side. Figure 3 illustrates this by a sketch of a two-dimensional RVE. The fiber marked by " 1 " protrudes beyond one edge of the RVE and is continued periodically on the opposite side. Furthermore, in the creation of the RVE, each fiber and each periodically shifted part of a fiber is checked for collision with other fibers. If a collision is detected, a new random position of the fiber is determined. The described procedure is carried out until all considered fibers are placed.

Following the geometry creation, the RVE is discretized by finite elements. The finite element mesh has a
Fig. 2 RVE reconstruction on the basis of a characterization and analysis of a real microstructure
Fig. $32 \mathrm{D}$ visualization of RVE with unidirectional fiber orientation and periodic structure. Virtual RVE's to illustrate the periodicity in light grey
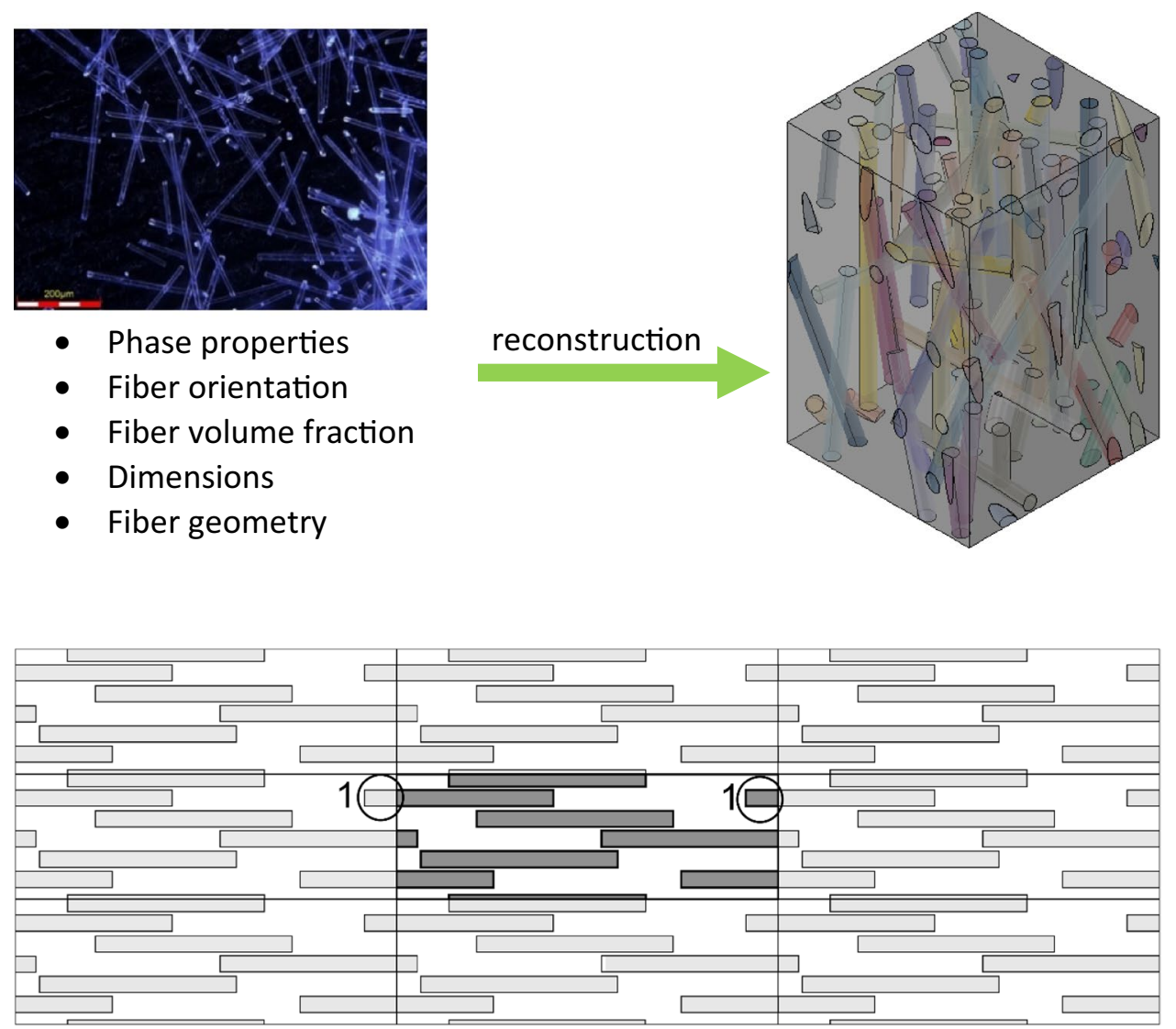

SN Applied Sciences A SPRINGER NATURE journal 
significant influence on the result of the finite element analysis. In this paper tetrahedral elements with a quadratic shape functions are used only.

Next, the creation of the periodic boundary conditions is presented. The periodic boundary conditions are defined on opposite surfaces of the RVE and connect them with each other. Considering a RVE with a periodic mesh, the mathematical description of the periodic boundary condition reads:

$u_{i}^{j+}-u_{i}^{j-}=\varepsilon_{i}\left(\boldsymbol{x}^{j+}-\boldsymbol{x}^{j-}\right)$

where $u$ denotes the displacement of opposite nodes on the surfaces $j+$ and $j-$ in the direction of $\varepsilon_{i}$ denotes the displacement of opposite points $x$ on the surface $j+$ and $j-$. The implementation of the periodic boundary conditions into a finite element analysis is carried out with linear constraint equations. Here the displacement $\varepsilon_{i}\left(x^{j+}-x^{j-}\right)$ is provided by a reference point:

$u_{i}^{j+}-u_{i}^{j-}+u_{i}^{R P}=0$

This reference point is not connected to the mesh of the RVE. Although the structure of the RVE is periodic, the finite element mesh may not be periodic. Opposite surfaces of the RVE are identical, but below the surface the geometries are different. Therefore, enforcing a periodic mesh simply by shifting of the nodes is not advantageous, since strongly distorted elements can result. Therefore, an interpolation approach is used instead that projects the periodic boundary conditions to a nonperiodic mesh. This publication uses an interpolation approach based on quadratic interpolation functions that is presented in the following.

Two opposite sides of the RVE mesh are being considered: For each node, with the coordinates $\boldsymbol{x}^{v}$, on one side of the RVE the surface of an opposite, enclosing element must be identified. If the nodes are virtually projected to a plane, the coordinates of the individual nodes are reduced to $\boldsymbol{x}^{v, r e d}$. These can be written in natural triangular coordinates $\zeta$ of the enclosing element with

$\left[\begin{array}{c}1 \\ x_{1}^{v, \text { red }} \\ x_{2}^{v, \text { red }}\end{array}\right]=\left[\begin{array}{ccc}1 & 1 & 1 \\ x_{1}^{1, \text { red }} & x_{1}^{2, \text { red }} & x_{1}^{3, \text { red }} \\ x_{2}^{1, \text { red }} & x_{2}^{2, \text { red }} & x_{2}^{3, \text { red }}\end{array}\right]\left[\begin{array}{c}\zeta_{1} \\ \zeta_{2} \\ \zeta_{3}\end{array}\right]$,

Here $x_{i}^{\prime}$ are the virtually reduced coordinates of the corner nodes of the enclosing element. With quadratic trial functions [22] and the natural triangle coordinates, the displacement of the node is interpolated with

$$
u_{i}^{-j}=\left[\begin{array}{llllll}
u_{i}^{1} & u_{i}^{2} & u_{i}^{3} & u_{i}^{4} & u_{i}^{5} & u_{i}^{6}
\end{array}\right]\left[\begin{array}{c}
\zeta_{1}\left(2 \zeta_{1}-1\right) \\
\zeta_{2}\left(2 \zeta_{2}-1\right) \\
\zeta_{3}\left(2 \zeta_{3}-1\right) \\
4 \zeta_{1} \zeta_{2} \\
4 \zeta_{2} \zeta_{3} \\
4 \zeta_{3} \zeta_{1}
\end{array}\right]
$$

In addition to the creation of the RVE's and the implementation of the periodic boundary condition, the homogenization method used is presented: both stresses and strains are integrated over the volume with

$\sigma^{*}=\frac{1}{V} \int_{V} \sigma d V$

or respectively

$\varepsilon^{*}=\frac{1}{V} \int_{V} \varepsilon d V$.

Due to the discretization already performed by the finite elements, the volume-integrals can be solved numerically with

$\sigma_{i j}^{*}=\frac{1}{V_{R V E}} \sum_{l=1}^{n_{i n t}} \sigma_{i j}^{l} \cdot V^{\prime}$

and

$\varepsilon_{i j}^{*}=\frac{1}{V_{R V E}} \sum_{l=1}^{n_{i n t}} \varepsilon_{i j}^{l} \cdot V^{\prime}$.

$V^{\prime}$ is the integration weight, which can be understood as the corresponding volume to the integration point.

In principle, the shown procedure can be carried out for each load direction (tension or compression in three spatial directions as well as shear in three spatial directions), so that the complete effective stiffness tensor can be analyzed. In this paper, only tensile deformation in the fiber direction ( $i=j=2)$ is applied, so that only the Young's modulus in fiber direction is analyzed:

$E_{22}=\frac{\sigma_{22}^{*}}{\varepsilon_{22}^{*}}$.

\section{Characterization}

The definition of the microstructure of a short fiber reinforced plastic requires knowledge about the fiber orientation and fiber length distribution as well as of the mechanical properties of the individual phases of the composite. A PBT-GF20 (Type 2300 GV1/20, 20 wt $\%$ glass fiber) from Celanex is used for all following investigations [23]. The 
mechanical material behavior of the glass fibers is considered to be isotropic, linear elastic with a Young's modulus $E_{f}=73.000 \mathrm{MPa}$ and a Poisson's ratio $v_{F}=0.22[24,25]$.

The mechanical behaviour of the matrix material PBT is tested by temperature-dependent mechanical tensile tests on bar-shaped specimens cut from $2 \mathrm{~mm}$ thick injection moulded plates in the dimensions $\mathrm{w}: 2 \mathrm{~mm}$, $\mathrm{d}: 3 \mathrm{~mm}, \mathrm{l}: 20 \mathrm{~mm}$. The mechanical behaviour of the composite material PBT-GF20 is also measured using the same specimen geometry. The fibers of the specimens are oriented in the longitudinal direction of the specimens. The tensile tests are carried out at $0{ }^{\circ} \mathrm{C}, 50^{\circ} \mathrm{C}$ and $80^{\circ} \mathrm{C}$, since these temperatures classify roughly the rubbery-, transition- and glassy regime of the PBT. The measured Young's moduli at these three temperatures of the PBT are $E_{M}=500 ; 1000$ and $2000 \mathrm{MPa}$ and $E_{C}=3050 ; 4500$ and $6800 \mathrm{MPa}$ for the PBT-GF20.

The fiber orientation is determined by a micro computed tomography $(\mu \mathrm{CT})$ measurement of a cylindrical specimen with a $1 \mathrm{~mm}$ radius, extracted out of the injection moulded plates. The voxel edge length measures $1.80 \mu \mathrm{m}$. The two-stage fiber orientation tensor $\boldsymbol{a}$ is determined on a cylindrical sample of an injection-molded plate [26]. The averaged fiber orientation tensor is represented by its eigenvalues eig $(\boldsymbol{a})=(0.244,0.711,0.045)$.

A discrete fiber orientation probability $\psi$ can be calculated according to Onat and Leckie [27] on the basis of the fiber orientation tensors. In addition to the measured second order fiber orientation tensor the fourth order fiber orientation tensor is approximated using the hybrid closure method [28]. Figure 4 shows the reconstructed orientation distribution on a unit sphere using a color plot. A

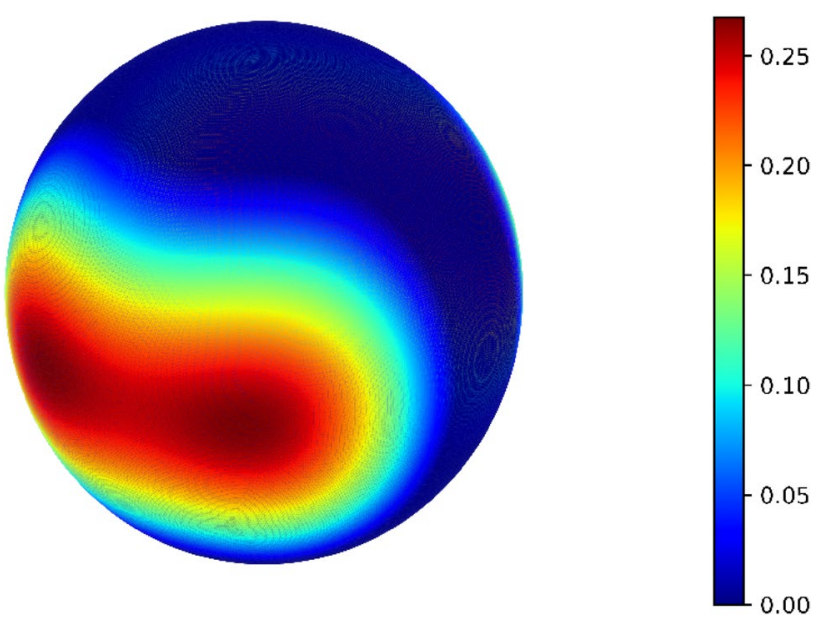

Fig. 4 Orientation distribution of injection molded specimen as color plot on a unit sphere. Recalculated by spherical harmonics with use of hybrid closure. Eigenvalues of $\boldsymbol{a}$ are $\operatorname{eig}(\boldsymbol{a})=(0.244,0.711,0.045)$ red color indicates a high probability that fibers are pointing in this direction.

The $\mu \mathrm{CT}$ can not be used to measure the fiber length distribution, as only fibers with an length exceeding $30 \mu \mathrm{m}$ can be detected due to the quality of detection of these fibers with the $\mu \mathrm{CT}$. The fibers shown in Fig. 5 are generated by incineration of a sample cut out of the injectionmolded plates. The fibers are mixed with ethanol and distributed on a Petri dish so that they are arranged as randomly as possible. Randomly selected sections of the Petri dish are then viewed under a light microscope and the length of the fibers is measured. The results in Fig. 5 proves that not negligibly few fibers are shorter than the $\mu C T$ resolution. The length distribution is determined by measuring 420 fibers from 3 different samples, which is shown in Fig. 6. A particularly high number of very short fibers can be seen here. The arithmetic mean is $172.7 \mu \mathrm{m}$,

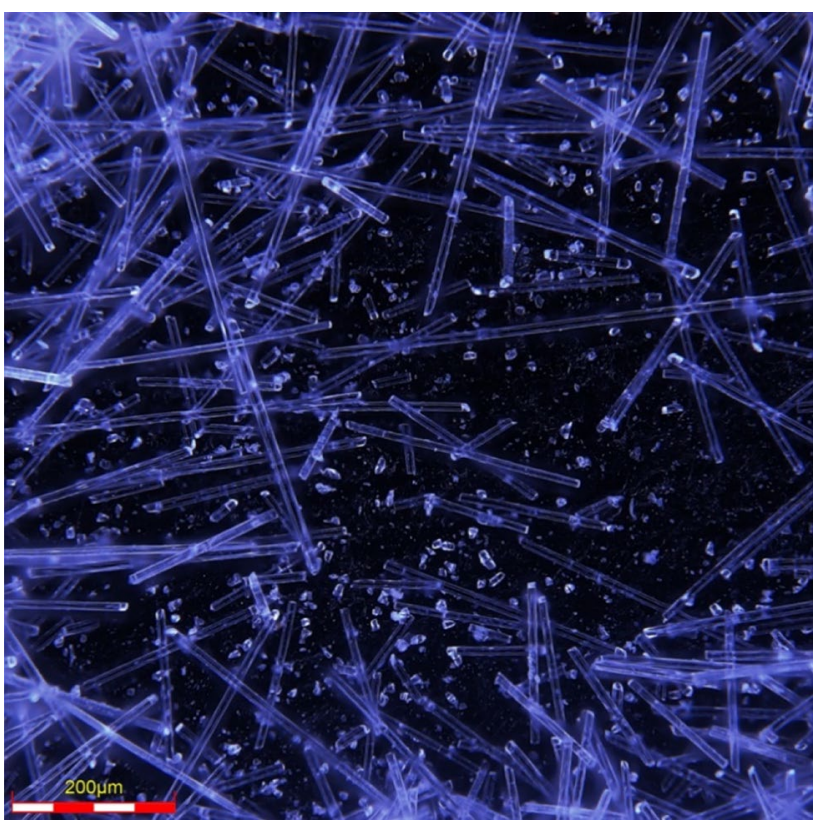

Fig. 5 Light microscopic image of exposed fibers from an ashed sample of PBT-GF20 to determine fiber length distribution

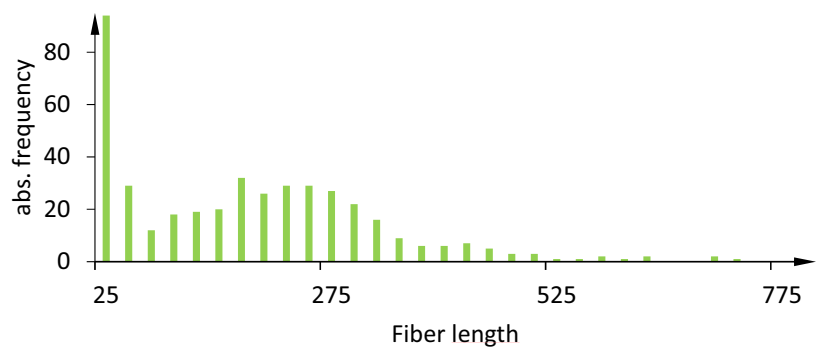

Fig. 6 Histogram of the fiber lengths of the exposed fibers from the injection molded sample 
which corresponds to an aspect ratio of 15.4 since the mean diameter of the fibers is 11.4 [26].

\section{Influence of modeling aspects}

This section examines the influence of modelling aspects. First, the influence of the fiber packing is investigated, followed by the fiber geometry. The influence of the fiber length distribution and the fiber orientation is then examined individually. Finally, the mentioned modeling aspects are combined. Each of the following finite element analysis is repeated with a refined mesh using $50 \%$ smaller element edges until a convergence of $1 \%$ deviation of the effective composite stiffness is reached.

\subsection{Influence of the fiber packing}

First, the influence of the fiber distance to each other is investigated using a unit cell. A unit cell is an RVE with a single fiber, where the position of the fiber can be random, but is always periodic. The influence of the distance between individual fibers can only be investigated in a unit cell with the use of periodic boundary conditions. With these, the mechanical behavior is identical to an infinitely extended RVE with homogeneous fiber distribution. The distance between the fibers is varied accordingly by changing the shape of the RVE. Figure 7 illustrates the arrangement of the unit cell. With the same volume and fiber volume content $\left(v_{F}=0.116\right)$, the distance between the fibers can be changed by varying the aspect ratio $a_{\text {RVE }}$. In the shown variant low RVE aspect ratio, the distance between the fibers is small in the direction of the fibers and large at right angles to them. In the high RVE aspect ratio variant, the distance in the fiber direction is larger than across it.

The change in the homogenized stiffness in the direction of the fibers is shown in Fig. 8. It can be seen that the stiffness decreases with increasing RVE aspect ratio. This effect is even more pronounced for the stiffer matrix material than for the less stiff matrix material. With a homogeneous fiber packing, i.e. when the distance $I=w$, the calculated effective composite stiffness by means of the RVE corresponds very accurate to that of the classical theory of Mori-Tanaka [3]. With a rheological equivalent model, the influence of the RVE aspect ratio can be explained by a simple series or
Fig. 7 Variation of RVE aspect ratio to vary distance of fibers with constant fiber size and constant fiber volume fraction
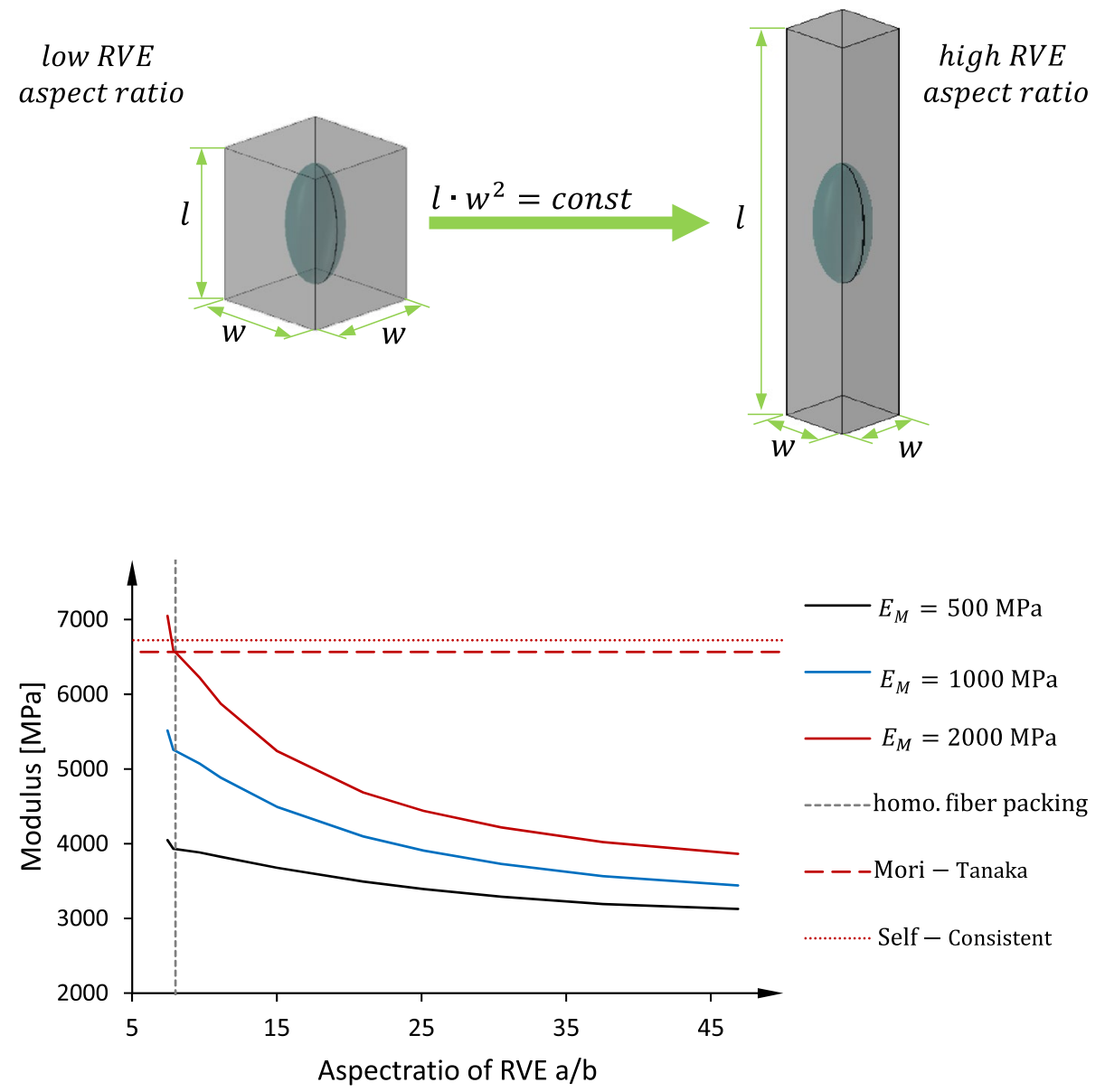
parallel connection of spring elements. If the aspect ratio of the RVE is large, the first approximation is a series connection of springs. If the aspect ratio is small, it is more likely that the springs are connected in parallel. Accordingly, the stiffness must be higher with a small aspect ratio of the RVE. The figure also shows that the effect of the fiber package is by no means negligible, as it has an extremely significant influence on the composite properties.

In the case of multiple fibers in an RVE, the package is no longer determined exclusively by the RVE dimension. In addition, the packing is determined by the arrangement within the RVE. The package, which is determined by the RVE dimension, is less important the more fibers are used in the RVE. This is illustrated by the mean distance between the fibers compared to the RVE dimension. In the case of several fibers, the mean value of the distance is $1 / n$ of the RVE dimension, where $n$ is the number of fibres. However, this means that the influence decreases with an increasing number of fibers, but remains significant for appropriate numbers of fibers $(<30)$. Therefore, the RVE dimension for $\mathrm{n}$ fibers of different lengths, diameters and orientation is determined in such a way that the mean distance between the fibers is identical in all spatial directions: First, the volume of the RVE $V_{R V E}$ is determined according to Eq. 1.

Then the RVE dimension is determined by an encapsulating rectangle for each fiber. The dimension of an encapsulating rectangle is identical to the maximum dimensions of the associated fibers, in the RVE's coordinate system. This means that a surrounding rectangle has surfaces parallel to the RVE. The dimensions of the enclosing rectangle is

$\mathbf{X}_{\mathrm{i}}=\left(\left|x_{i, \max }-x_{i, \min }\right|,\left|y_{i, \max }-y_{i, \text { min }}\right|,\left|z_{i, \max }-z_{i, \min }\right|\right)$.

with $x, y, z$ as the minimal or maximal coordinates of the fibers. From the encapsulating rectangles a middle encapsulating rectangle with

$\mathbf{X}_{\mathrm{F}}=\frac{\sum_{i=1}^{n} \mathbf{X}_{i}}{n}$

is calculated. The dimension of the RVE is now determined by a constant offset $s$ to this middle encapsulating rectangle with

$\mathbf{X}_{\mathrm{RVE}}=\boldsymbol{X}_{F}+s$

For the offset $s$, the condition that the product of the dimension of the RVE is equal to the volume of the RVE applies:

$\prod_{i=1}^{3}\left(X_{i}+s\right)=\mathbf{V}_{\mathrm{RVE}}$

This cubic equation can be solved either analytically or numerically, for example using the Newton method.
Among the three possible mathematical solutions only one is a physically feasible solution. This one solution is not a complex solution.

\subsection{Fiber shape}

Next, the influence of the geometric shape of the fibers on the effective composite behavior is investigated. For this purpose, the stiffness of the composite in relation to the aspect ratio of the fiber is examined using a unit cell with one fiber and homogeneous fiber packing. The fiber volume fraction is constant with $v_{F}=0.116$. The fibers are modeled as ellipsoids and as cylinders. In the case of fibers represented by cylinders, a distinction is made between a matrix fully tied to the fiber and a matrix tied to the lateral surface of the fiber only. This strategy is motivated by the fiber sizing. The sizing serves as an adhesion agent between matrix and fiber and is applied during the manufacturing process of endless fibers before they are cut to short fibers. In the injection molding process, the fibers break further into smaller pieces. Accordingly, there is no sizing on the fiber's cross section surfaces, leading to a poor adhesion between fiber and matrix at these positions.

The Fig. 9 shows the result of the investigation regarding the influence of the geometric shaping of the fibers. One can state, that in the case of cylindrical fiber reinforcements and a matrix fully tied to the fibers the homogenized composite stiffness is independent of the matrix stiffness greater than that of the ellipsoid fiber reinforcement. This effect is almost repeatable for aspect ratios of the fibers from 1 to 30 . The composite stiffness using a matrix that is tied to the lateral surface of the cylindrical fibers only ranges between the composite stiffness of ellipsoid and fully bonded fibers.

\subsection{Length distribution}

If more than one fiber is used for the reconstruction of the microstructure, two concepts regarding the fiber length can be adopted in principle. On the one hand, all fibers can be modeled of the same length equals the average fiber length. The mean fiber length can be defined, for example, by the arithmetic mean or the median. On the other hand, a fiber length distribution can be applied. The difference between the two concepts in terms of homogenized composite stiffness will be analyzed in the following study. For this purpose, both concepts are applied and compared. For the concept of mean fiber length, the arithmetic mean value of the fiber length distribution measured in Sect. 3 is used as the mean length. In the concept of fiber length distribution, the measured fiber lengths are divided into 
Fig. 9 Effective modulus in direction of fiber reinforcement calculated by RVE model for different matrix modulus to determine the influence of the geometrical modeling of the fibers
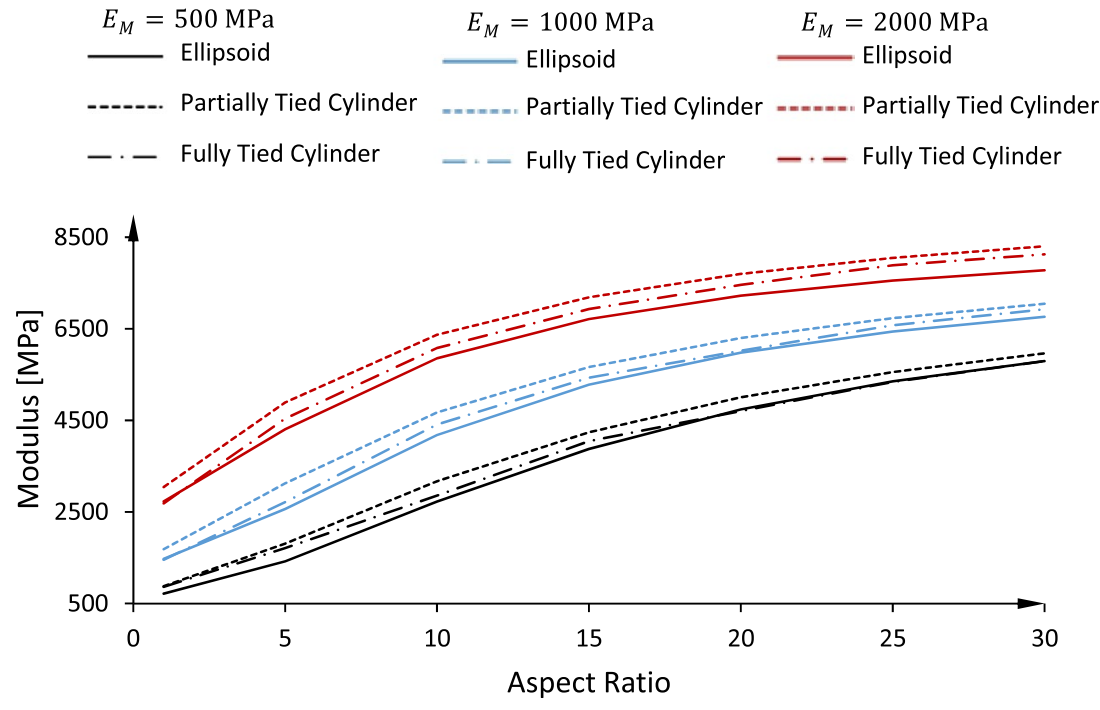

classes (see Fig. 6) of $25 \mu \mathrm{m}$ width. For each class, a probability is determined according to the number of fibers within the class. This probability is used to reconstruct the microstructure so that the same fiber length distribution is obtained with a statistically sufficient number of reconstructed fibers. For both concepts the constant fiber volume content $v_{F}=0.116$ and a matrix stiffness $E_{M}=2000 \mathrm{MPa}$ are identical. All fibers are distributed randomly in space. Accordingly, a Monte-Carlo simulation must be carried out for both concepts in this investigation. Figure 10 shows the implementation of the Monte Carlo simulations. First $n$ RVE's are generated and the effective stiffness of each is determined by finite element analyses and the homogenization scheme described in Sect. 2. A probability distribution is then determined from the calculated values. In contrast to the unit cell with a single fiber, the result depends on the spatial position of the fibers in the RVE due to fiber interacting. Additionally, the Monte-Carlo simulation is necessary for the fiber length probability, since reconstructions with few fibers do not provide a good approximation to the measured fiber length distribution. This means that the reconstruction is carried out several times (min. 100 times) for both concepts. Both concepts are repeated for different numbers of fibers. The result of the study is shown in Fig. 11.

The normal distributions of the calculated effective stiffness of the composite in fiber direction are shown for both concepts and 10,30 and 50 fibers respectively. The mean values of both concepts converge to different values. For a uniform length distribution, the mean value for 50 fibers is approximately $6500 \mathrm{MPa}$, using a fiber length distribution it is approximately $7600 \mathrm{MPa}$. The mean value of the effective stiffness is thus significantly higher with fiber length distribution than with uniform fiber length. In both concepts, the mean values with 10,30 and 50 fibers are approximately identical, with the exception of 10 fibers in the case of the fiber length distribution. In this case the dimensions of the RVE are smaller than some fibers. Therefore, the RVE may not be modelled as short fibers, but as endless fibers. This leads to a higher mean value of the effective composite stiffness. In addition, it becomes apparent that the standard deviation of the effective composite stiffness is greater with the concept of fiber length distribution than with a uniform fiber length, because it contains another random variable. Accordingly, the convergence rate is lower when using the fiber length distribution, so that more RVE's have to be considered in order to determine the stiffness with the same confidence level.
Fig. 10 Illustration of the Monte Carlo simulation. Creation of $n$ RVE's with subsequent homogenization and derivation of the probability function of the effective Modulus

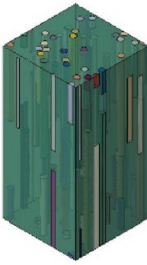

RVE 1
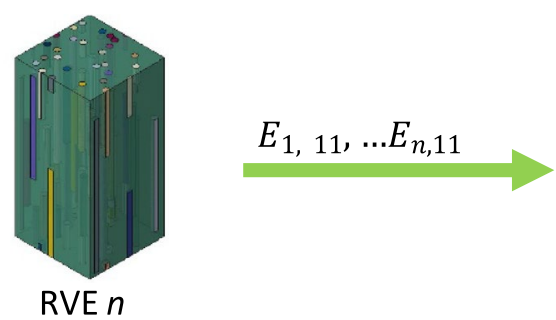

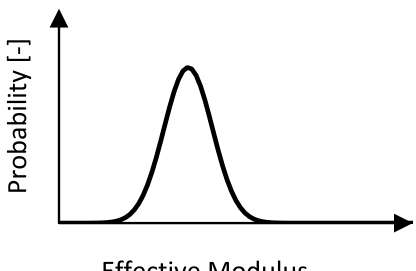

Effective Modulus 
Fig. 11 Normal Gaussian distribution of effective composite E-modulus $E_{11}$ in direction of fiber reinforcement for 10, 30 and 50 with equal fiber lengths and applied fiber length distribution

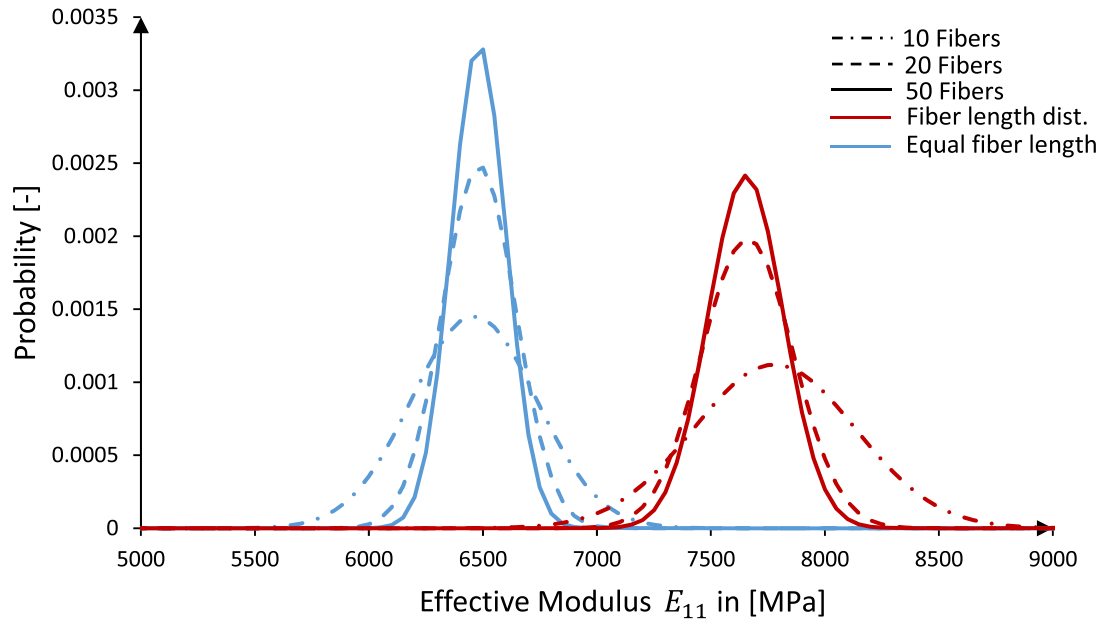

Another important aspect becomes also evident with the distribution of the homogenized composite stiffness. A higher homogenized stiffness means that the local stresses within such an RVE are higher with the same deformation than the local stresses within RVE's which lead to a lower homogenized stiffness. The local stresses are therefore dependent on the fiber arrangement and are statistically depended variable.

\subsection{Fiber orientation}

Finally, the influence of fiber orientation on the effective composite stiffness is investigated. The focus is particularly on fiber orientations of small angles between the fibers and the "flow" direction. This is because experimental material characterization tests are often carried out on injection molded specimens with a confined fiber orientation, but of course, these specimens do not feature a perfectly unidirectional fiber orientation. Figure 12 shows the principle RVE design for the investigation of this modelling aspect. The RVEs reconstructed for this study use a constant fiber volume ratio $v_{f}=0.116$ and 20 fibers of the same length of $172.7 \mu \mathrm{m}$. The polar angle $\varphi$ of the fibers varies in $5^{\circ}$ steps, while the azimuth angle $\theta$ of each fiber is random between 0 and $360^{\circ}$. Each angular step is performed with 20 repetitions of the reconstruction, so that the calculated effective composite stiffness is statistically reliably evaluated. Figure 13 shows the result. For a matrix stiffness of $E_{M}=2000 \mathrm{MPa}$, the effective composite stiffness is maximum for an angle of $0^{\circ}$ and decreases with increasing angle. For lower stiffnesses the maximum is shifted towards a small angle, but then also decreases for even larger angles.

\subsection{Combination of modelling aspects}

Up to now, the influence of four different modelling aspects on the effective composite stiffness has been investigated individually. In the following, the modeling aspects are considered in combination by reconstructing the characterized composite PBT-GF20. This means, that the measured fiber length distribution (see Fig. 6) and the reconstructed fiber orientation (see Fig. 4) are used for the RVE. Cylindrical fibers are considered where the matrix is tied at the fiber's lateral surfaces. The combined modeling aspects indicate how precisely the effective composite properties can be determined. Figure 14 shows the normal distribution of the calculated effective composite stiffness in the fiber direction with combined modeling aspects. It can be conducted that the mean value converges to
Fig. 12 Variation of fiber orientation for small angles with the same volume fraction and aspect ratio of fibers
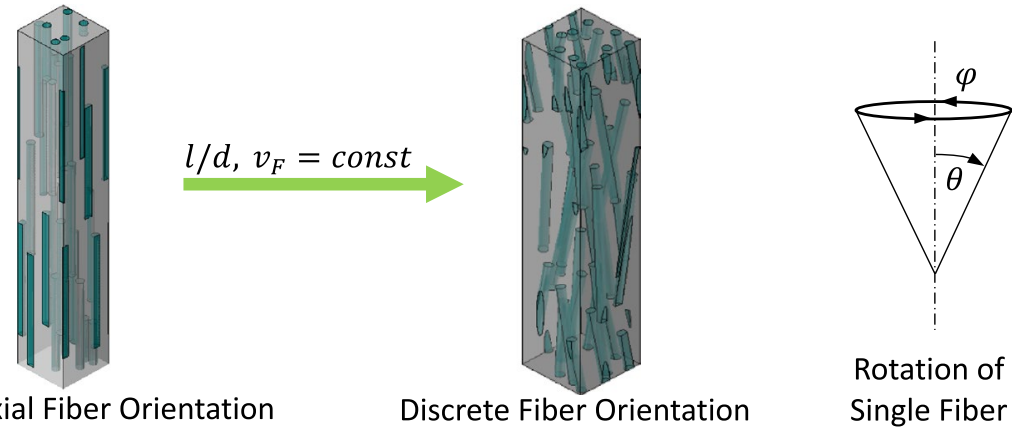

Rotation of Single Fiber 

direction of fiber reinforcement calculated by RVE model for a discrete fiber orientation depending on the E-modulus of the matrix material
Fig. 13 Effective modulus in

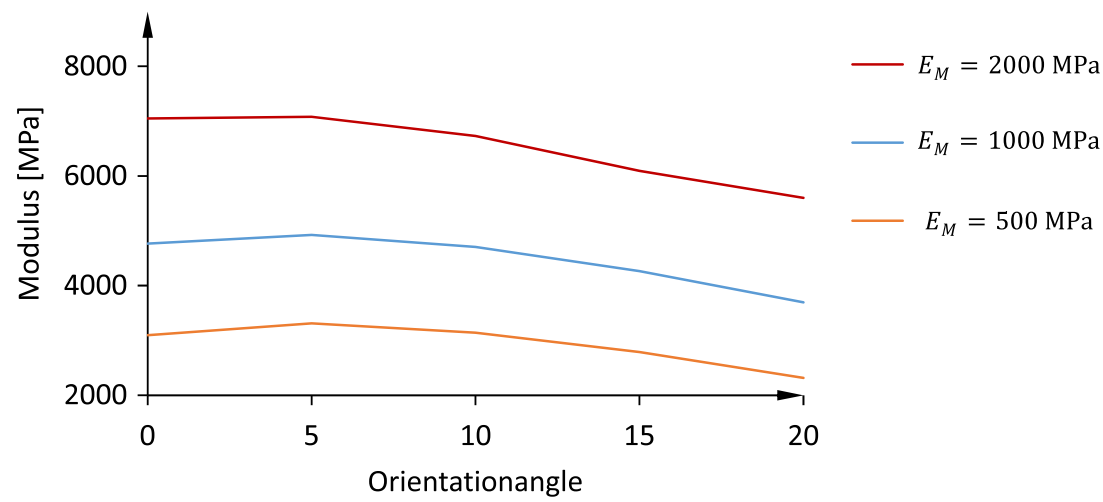

Fig. 14 Normal Gaussian distribution of effective composite E-modulus $E_{11}$ in direction of fiber reinforcement for 10,30 and 50 fiber with equal fiber lengths, applied fiber length distribution and combined modelling parameters

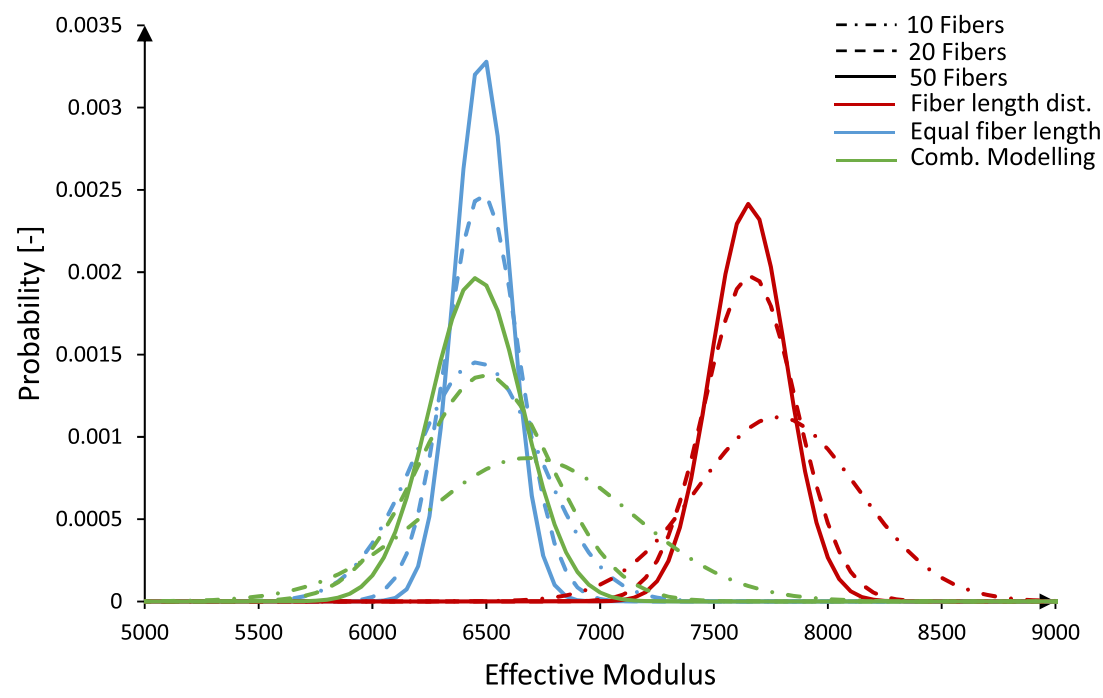

approx. $6450 \mathrm{MPa}$ with the consideration of 50 fibers. Obviously, the standard deviation is greater than for the investigations with uniform length or with fiber length distribution.

\section{Discussion}

In this section, a discussion of the investigated modelling aspects follows. The fiber package in short fiber reinforced plastics has a large influence on the resulting effective composite properties. For a unit cell with one fiber, the fiber package is only determined by the dimension of the RVE and its influence is principally studied frequently in the literature [11,29-32]. With many fibers, in the sense of the definition of an RVE according to Hill the fiber packing is not effected by the dimensions of the RVE. With only a few fibers, however, as for the Drugan and Willis approach to determine an RVE as the smallest possible volume, the fiber package depends both on the realized arrangement and on the dimension. As shown in this paper by the investigation of the influence of the fiber package, it is very significant for the effective composite stiffness of short fiber reinforced plastics. Therefore, it is important to model the fiber package accurately for a precise prediction of real composite materials. For the modelling of a RVE in the sense of the Drugan and Willis approach, it seems to be reasonable to determine the dimension of the RVE in such a way that a homogeneous fiber package results on average with the Monte-Carlo approach, which can be achieved with the presented approach in Sect. 4.1.

Using the Monte-Carlo approach with many fibers (unidirectional and equal length), the mean value of the effective stiffness corresponds to that of a homogeneous fiber package (see Figs. 9, 11). It is important to note, however, that this only applies to the average stiffness. The effective stiffness of a single RVE always depends on the fiber constellation realized. As the size of the RVE increases, it is only more likely to produce a fiber constellation that corresponds to a homogeneous fiber packing.

The dependence of the aspect ratio on the effective composite stiffness with ellipsoidal fibers is well known in the literature from the model of Mori-Tanaka [3]. Qualitatively, this dependence is the same for the fully and 
partially bonded cylindrical fibers, as shown in this study. Thereby the fully bonded cylinders results in higher effective composite stiffness compared to ellipsoids, while the partially bonded are in-between. The difference between fully and partially bonded decreases with increasing aspect ratio. The overall influence of the fiber shape and bondage on the effective composite stiffness can therefore be classified as medium, especially for aspect ratios which typically occur in technical applications. However, it remains to be investigated to what extent this is also the case in the calculation of the strength of the composite.

The results of the investigation with the combination of the different modelling aspects are also compared with experimental results of a characterization of PBT-GF20. For this purpose, the converged mean value of the calculated stiffness by the RVE is used. In addition, the calculated stiffness using the Mori-Tanaka [3] approach, the approach of Duschelbauer [33] and the self-consistent [34] method are used as an additional comparison. To consider the effects of fiber orientation and fiber length distribution, a twostep homogenization is used.

Figure 15 shows the results of the effective composite stiffness in fiber direction. The RVE's method calculate the effective composite stiffness with a very small deviation. In contrast, the calculated stiffness's of the three comparison models deviate significantly from the experimental results. Although ellipsoidal inclusions are used in each of the three comparison models to model the fiber's geometry, it can be shown (cf. Fig. 9) that ellipsoidal geometry cannot be the exclusive cause of the deviation between calculated and measured stiffness. As the self-consistent method has a higher prediction quality than the two other comparison models, it is evident, that the influence of the fibers on each other cannot be neglected for the fiber volume fraction used here. Nevertheless, due to the two-step homogenization method with the self-consistent method, no fiber influence can be performed across fiber classes of the two-step homogenization. With RVE's there is no such disadvantage, in principle every modeling aspect can interact with others.

\section{Conclusion}

This paper investigates the influence of four modelling aspects on the effective stiffness of a short-fiber-reinforced plastics. For this purpose, RVEs are reconstructed on the basis of characteristic quantities determined experimentally. The influence of the four modeling aspects on the effective composite stiffness are determined by variation. The four modeling aspects include fiber packing, fiber shape, fiber length distribution and fiber orientation.

- The fiber package has a very significant influence on the calculated homogeneous composite stiffness. This can be conducted by an RVE with an elliptical fiber and varied RVE dimensions. A homogeneous fiber package, i.e. the distance in all directions is the same, reproduces the results of the Mori-Tanaka model.

- The fiber shape has a medium influence on the homogeneous composite stiffness. Cylindrical fibers are stiffer than ellipsoid fibers with the same fiber volume content and aspect ratio. Fibers with a matrix tied on their lateral surface only owns a homogenized stiffness between the stiffness of cylindrical and ellipsoid modelled fibers.

- A fiber length distribution influences the effective stiffness compared to a constant average fiber length. In addition to the mean value of the stiffness, the scattering width of the effective stiffness is higher.

- The combined consideration of modeling aspects in the RVE provides a very accurate prediction of a composite's effective stiffness, in the case of the used PBTGF20 for the validation more accurate than the MoriTanka-, Duschelbauer- or Self-Consistent Method [3, 33, 34].
Fig. 15 Results of effective composite stiffness by experiment, RVE model, self-consistent method, Mori-Tanaka and Duschlbauer [3, 33, 34]

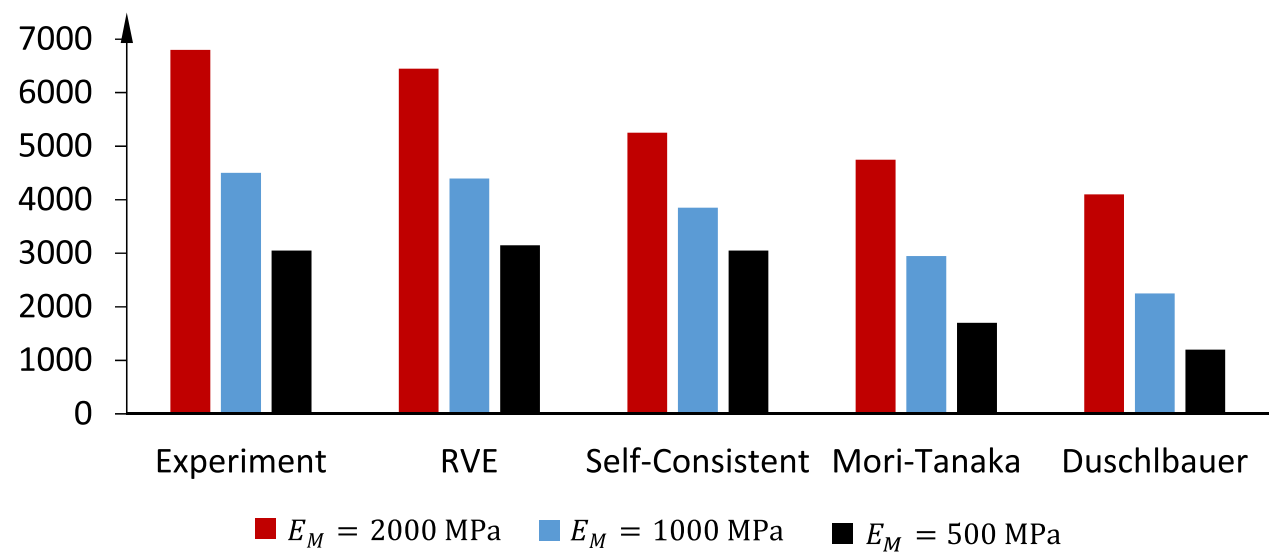

SN Applied Sciences A SPRINGER NATURE journa 
From the results of the numerical investigations it can be concluded that a precise knowledge of the fiber packing, fiber length distribution, fiber orientation and fiber geometry is essential for a precise determination of the effective composite properties.

In further studies it has to be clarified to what extent the influence of the modelling parameters remains the same for other load directions as well as for a plastic or viscoplastic modelling of the matrix material.

\section{Compliance with ethical standards}

Conflict of interest On behalf of all authors, the corresponding author states that there is no conflict of interest.

Open Access This article is licensed under a Creative Commons Attribution 4.0 International License, which permits use, sharing, adaptation, distribution and reproduction in any medium or format, as long as you give appropriate credit to the original author(s) and the source, provide a link to the Creative Commons licence, and indicate if changes were made. The images or other third party material in this article are included in the article's Creative Commons licence, unless indicated otherwise in a credit line to the material. If material is not included in the article's Creative Commons licence and your intended use is not permitted by statutory regulation or exceeds the permitted use, you will need to obtain permission directly from the copyright holder. To view a copy of this licence, visit http://creativecommons .org/licenses/by/4.0/.

\section{References}

1. Reuss A (1929) Berechnung der Fließgrenze von Mischkristallen auf Grund der Plastizitätsbedingung für Einkristalle. Z Angew Math Mech. https://doi.org/10.1002/zamm.19290090104

2. Voigt W (1889) Ueber die Beziehung zwischen den beiden Elasticitätsconstanten isotroper Körper. Ann Phys. https://doi. org/10.1002/andp.18892741206

3. Mori T, Tanaka K (1973) Average stress in matrix and average elastic energy of materials with misfitting inclusions. Acta Metall. https://doi.org/10.1016/0001-6160(73)90064-3

4. Hill R (1963) Elastic properties of reinforced solids. Some theoretical principles. J Mech Phys Solids. https://doi.org/10.1016/00225096(63)90036-X

5. Drugan WJ, Willis JR (1996) A micromechanics-based nonlocal constitutive equation and estimates of representative volume element size for elastic composites. J Mech Phys Solids. https:// doi.org/10.1016/0022-5096(96)00007-5

6. Gitman IM, Askes H, Sluys LJ (2007) Representative volume. Existence and size determination. Eng Fract Mech. https://doi. org/10.1016/j.engfracmech.2006.12.021

7. Huet C (1990) Application of variational concepts to size effects in elastic heterogeneous bodies. J Mech Phys Solids. https://doi. org/10.1016/0022-5096(90)90041-2

8. Hazanov S, Huet C (1994) Order relationships for boundary conditions effect in heterogeneous bodies smaller than the representative volume. J Mech Phys Solids. https://doi. org/10.1016/0022-5096(94)90022-1

9. Kanit T, Forest S, Galliet I, Mounoury V, Jeulin D (2003) Determination of the size of the representative volume element for random composites: statistical and numerical approach. Int J Solids Struct. https://doi.org/10.1016/S0020-7683(03)00143-4

10. Gusev AA (1997) Representative volume element size for elastic composites: a numerical study. J Mech Phys Solids. https://doi. org/10.1016/S0022-5096(97)00016-1

11. Wang Z, Smith DE (2019) Numerical analysis on viscoelastic creep responses of aligned short fiber reinforced composites. Compos Struct. https://doi.org/10.1016/j.compstruct .2019 .111394

12. Babu KP, Mohite PM, Upadhyay CS (2018) Development of an RVE and its stiffness predictions based on mathematical homogenization theory for short fibre composites. Int J Solids Struct. https://doi.org/10.1016/j.ijsolstr.2017.10.011

13. Berger $\mathrm{H}$, Kari S, Gabbert U, Rodríguez-Ramos R, Bravo-Castillero J, Guinovart-Díaz R, Volume D (2007) No: evaluation of effective material properties of randomly distributed short cylindrical fiber composites using a numerical homogenization technique. J Mech Mater Struct. https://doi.org/10.2140/jomms .2007.2.1561

14. Chen L, Gu B, Tao J, Zhou J (2019) The average response and isotropy of 3D representative volume elements for random distributed short fibers reinforced elastomer. Compos Struct. https ://doi.org/10.1016/j.compstruct.2019.02.049

15. Chen L, Gu B, Zhou J, Tao J (2019) Study of the effectiveness of the RVEs for random short fiber reinforced elastomer composites. Fibers Polym. https://doi.org/10.1007/s12221-019-1178-9

16. Pan Y, lorga L, Pelegri AA (2008) Analysis of 3D random chopped fiber reinforced composites using FEM and random sequential adsorption. Comput Mater Sci. https://doi.org/10.1016/j.comma tsci.2007.12.016

17. Pan $Y$, lorga $L$, Pelegri AA (2008) Numerical generation of a random chopped fiber composite RVE and its elastic properties. Compos Sci Technol. https://doi.org/10.1016/j.compscitec h.2008.06.007

18. Burgarella B, Maurel-Pantel A, Lahellec N, Bouvard J-L, Billon N, Moulinec H, Lebon F (2019) Effective viscoelastic behavior of short fibers composites using virtual DMA experiments. Mech Time Depend Mater. https://doi.org/10.1007/s11043-018-9386-z

19. Wang L, Nygren G, Karkkainen RL, Yang Q (2019) A multiscale approach for virtual testing of highly aligned short carbon fiber composites. Compos Struct. https://doi.org/10.1016/j.comps truct.2019.111462

20. Okereke MI, Akpoyomare Al (2013) A virtual framework for prediction of full-field elastic response of unidirectional composites. Comput Mater Sci. https://doi.org/10.1016/j.comma tsci.2012.12.036

21. Nguyen V-D, Béchet E, Geuzaine C, Noels L (2012) Imposing periodic boundary condition on arbitrary meshes by polynomial interpolation. Comput Mater Sci. https://doi.org/10.1016/j. commatsci.2011.10.017

22. Advanced Finite Element Methods (ASEN 6367) Course Material. https://www.colorado.edu/engineering/CAS/courses.d/ AFEM.d/. Accessed 16 Feb 2018

23. Celanese (2017) Celanex 2300 GV1/20 Datasheet. http://catal og.ides.com/Datasheet.aspx?I=26793\&E=73487. Accessed 12 July 2017

24. Kaiser J-M, Stommel M (2014) Modified mean-field formulations for the improved simulation of short fiber reinforced thermoplastics. Compos Sci Technol. https://doi.org/10.1016/j.comps citech.2014.05.010

25. Parra-Venegas EJ, Campos-Venegas K, Martinez-Sanchez R, Herrera-Ramirez JM, Rodriguez-Miranda A (2012) The tensile behavior of E-glass fibers. Microsc Microanal. https://doi.org/10.1017/ S1431927612005776 
26. Kaiser J-M (2013) Beitrag zur mikromechanischen Berechnung kurzfaserverstärkter Kunststoffe-Deformation und Versagen. Dissertation, Universität des Saarlandes

27. Leckie FA, Onat ET (1981) Tensorial nature of damage measuring internal variables. In: Hult J, Lemaitre J (eds) Physical nonlinearities in structural analysis. Springer, Berlin, pp 140-155

28. Advani SG, Tucker CL (1987) The use of tensors to describe and predict fiber orientation in short fiber composites. J Rheol. https ://doi.org/10.1122/1.549945

29. Swolfs Y, Verpoest I, Gorbatikh L (2016) A review of input data and modelling assumptions in longitudinal strength models for unidirectional fibre-reinforced composites. Compos Struct. https://doi.org/10.1016/j.compstruct.2016.05.002

30. Landis CM, McMeeking RM (1999) Stress concentrations in composites with interface sliding, matrix stiffness and uneven fiber spacing using shear lag theory. Int J Solids Struct. https://doi. org/10.1016/S0020-7683(98)00193-0

31. Sun CT, Vaidya RS (1996) Prediction of composite properties from a representative volume element. Compos Sci Technol. https://doi.org/10.1016/0266-3538(95)00141-7
32. Bargmann S, Klusemann B, Markmann J, Schnabel JE, Schneider K, Soyarslan C, Wilmers J (2018) Generation of 3D representative volume elements for heterogeneous materials: a review. Prog Mater Sci. https://doi.org/10.1016/j.pmatsci.2018.02.003

33. Duschlbauer D, Pettermann HE, Böhm HJ (2003) Mori-Tanaka based evaluation of inclusion stresses in composites with nonaligned reinforcements. Scripta Mater. https://doi.org/10.1016/ S1359-6462(02)00390-1

34. Hill R (1965) A self-consistent mechanics of composite materials. J Mech Phys Solids. https://doi.org/10.1016/00225096(65)90010-4

Publisher's Note Springer Nature remains neutral with regard to jurisdictional claims in published maps and institutional affiliations. 